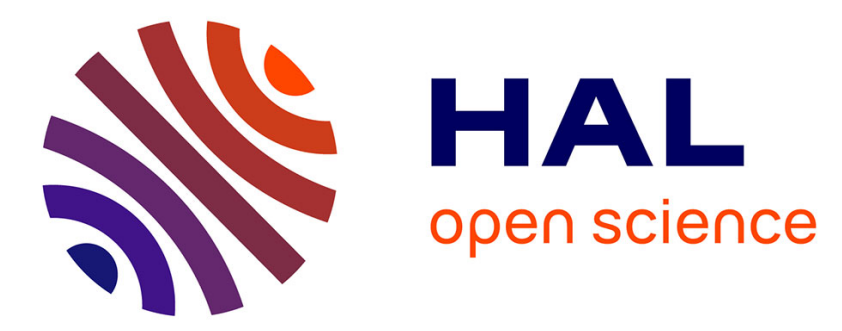

\title{
Modelling Data Processing for Interactive Scores Using Coloured Petri Nets
}

\author{
Jaime Arias, Myriam Desainte-Catherine, Camilo Rueda
}

\section{To cite this version:}

Jaime Arias, Myriam Desainte-Catherine, Camilo Rueda. Modelling Data Processing for Interactive Scores Using Coloured Petri Nets. 14th International Conference on Application of Concurrency to System Design, Jun 2014, Tunis, Tunisia. 10.1109/ACSD.2014.23 . hal-01095176

\section{HAL Id: hal-01095176 https://hal.science/hal-01095176}

Submitted on 22 Sep 2015

HAL is a multi-disciplinary open access archive for the deposit and dissemination of scientific research documents, whether they are published or not. The documents may come from teaching and research institutions in France or abroad, or from public or private research centers.
L'archive ouverte pluridisciplinaire HAL, est destinée au dépôt et à la diffusion de documents scientifiques de niveau recherche, publiés ou non, émanant des établissements d'enseignement et de recherche français ou étrangers, des laboratoires publics ou privés. 


\section{Modelling Data Processing for Interactive Scores Using Coloured Petri Nets}

\author{
Jaime Arias, Myriam Desainte-Catherine \\ Univ. Bordeaux, LaBRI, Bordeaux, F-33000, France \\ CNRS, UMR 5800, Bordeaux, F-33000, France \\ IPB, LaBRI, Bordeaux, F-33000, France \\ Email: \{jaime.arias, myriam\}@labri.fr
}

\author{
Camilo Rueda \\ Departamento de Electrónica y \\ Ciencias de la Computación \\ Pontificia Universidad Javeriana \\ Cali, Colombia \\ Email: crueda@javerianacali.edu.co
}

\begin{abstract}
I-score is a system for the composition and execution of interactive multimedia scores. It uses Hierarchical Time Stream Petri Nets (HTSPN) to build an execution model of the scores. Nowadays, composers have increasingly needed to represent and manipulate complex data in their multimedia scenarios. However, HTSPN formalism does not allow to handle data. In this work, we propose a model to execute interactive multimedia scores based on Coloured Petri Nets (CPN). Our work extends the current execution model of $i$-score with the capability to handle complex data. Our approach consists in developing CPN modules for reading, appending and reversing audio files. We use CPN Tools for prototyping, simulating and verifying our model and discuss how to represent fundamental signal processing functions, conditionals or loops.
\end{abstract}

Keywords—coloured petri nets; data processing; formal specification; interactive scores;

\section{INTRODUCTION}

Nowadays, the design of interactive multimedia systems based on a written scenario is a challenge that requires to handle dynamic and static events as well as dynamic and static data. Interactive scores [1] propose a model to write and execute interactive scenarios comprised of several multimedia processes. This model provides a real-time control of the dynamic and static events, and also it preserves the temporal organization of the scenario during the writing and execution stage.

I-SCORE [2] is a software that allows to design and execute interactive and multimedia scenarios. The execution model is based on Hierarchical Time Stream Petri Nets (HTSPNs) [3]. Places and transitions of Petri nets model temporal aspects of the scenario (i.e., they define a partial order between static and dynamic events) defined during its composition.

This paper presents an extension of I-SCORE that aims to handle: (1) complex data, in particular, dynamic and static data streams; and (2) dynamic and static events. The extension adds the possibility of building stream processing structures by functional composition of processes through input/output data slots. Our main idea is to use Coloured Petri Nets (CPNs) [4] to model complex data and the dynamic aspect of the functional composition of processes. Multimedia streams are often cut into temporal frames to be carried from one process to another. Then, in our approach, we model frames as coloured tokens that are handled by processes.
The extension we propose provides the notion of asynchronous functional composition. This corresponds to the case where the composed processes are not executed at the same time. Then, it requires to buffer the output data stream of processes in order to hold data until another process read them. In the context of interactive scores, the date in which the buffers will be read may not be known at composition nor execution time. Nevertheless, the duration of the buffers are all bounded by the duration of the scenario which is finite. Therefore, we are very interested in verifying properties of scenarios by using the verification methods of CPNs.

The rest of the paper is organized as follows. In Section II we briefly present the I-SCORE system and the Coloured Petri Nets formalism. In Section III we develop a model for executing interactive scores using Coloured Petri Nets. In Section IV, we extend our model for handling complex data. Finally, in Section V we present conclusions, and future work.

\section{PRELIMINARIES}

In this section we first briefly present the I-SCORE system. Then, we present the basic notions of Coloured Petri Nets (CPNs), and we also show a tool for editing, simulating and verifying CPNs models.

\section{A. I-score}

I-SCORE is a software for the composition and execution of interactive multimedia scores [1], [2]. It consists of two sides: authoring side and performance side. The authoring side allows the composer to design a scenario. On the other hand, the performance side executes the scenario in real-time with interactive capabilities.

1) Authoring side: In this side the composer designs a scenario as a collection of multimedia objects that are represented as boxes on a sheet in which the horizontal axis represents the time-line and the vertical axis has no meaning. The boxes are temporal structures with a start date, a duration and a multimedia process. In I-SCORE, the execution of the multimedia processes is carried out by external applications such as MAX/MSP ${ }^{1}$ or PURE DATA ${ }^{2}$, and it uses multimedia protocols such as $\mathrm{OSC}^{3}$ to send values/parameters to them.

\footnotetext{
${ }^{1}$ http://cycling74.com/products/max/

${ }^{2} \mathrm{http}: / /$ puredata.info

${ }^{3} \mathrm{http}: / /$ opensoundcontrol.org
} 
The composer can use hierarchical boxes for grouping several boxes with their own temporal organization. Hierarchical boxes eases the design of large scenarios. In fact, the whole scenario is represented as a hierarchical box.

The composer partially defines the temporal organization of the scenario using Allen temporal relations [5] between the boxes. These represent temporal precedences between boxes, that are complemented with quantitative measures in the form of durations. It is possible thus to express that the start of some box must be separated from the end of some other by a given duration. I-SCORE allows composers to build interactive scores adding discrete interactive events which are triggered in execution time and modify either the start date or the duration of the boxes. It is important to note that temporal relations are preserved during the authoring and performance sides. For this reason, a scenario can be interpreted in a limited set of possibilities.

In Figure 1, we show an interactive scenario where: the box A has no interaction points, then it has a fixed start date and duration; the box $\mathrm{C}$ has an interaction point at the start, then the start date can be modified in execution time; the box D has an interaction point at the end, then the duration of the box can be modified in execution time; and the box B combines the two above behaviours. Note that the temporal relations preceding the boxes $\mathrm{B}$ and $\mathrm{C}$ are represented as dashed arrows, it means that the duration of the temporal relation is partially defined by a range of possible values. Otherwise, the duration of the interval is fixed and it is represented as solid arrows.

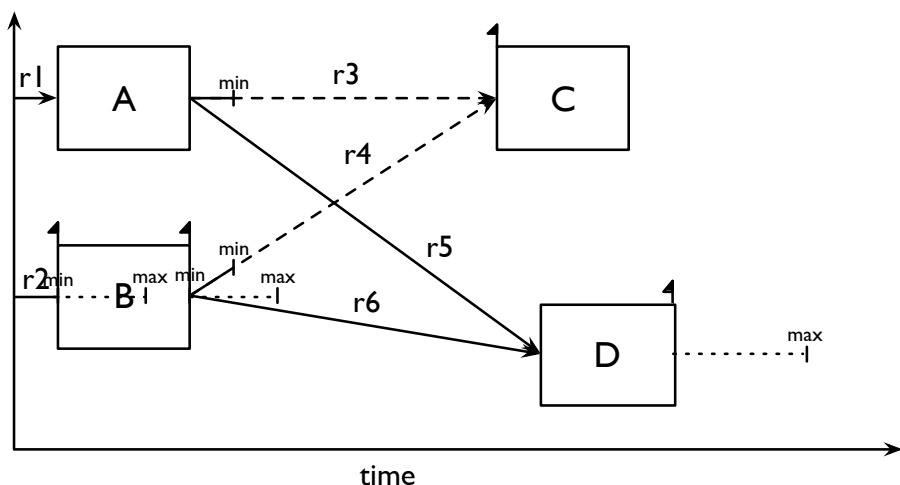

Fig. 1. Example of an interactive scenario.

2) Performance side: I-SCORE translates the scenario into a Hierarchical Time Stream Petri Net (HTSPN) [3] structure for its execution. The equivalent HTSPN structure allows to trigger interactive events, and also preserves the temporal organization during the execution of the score. The method for transforming a score into a HTSPN structure is described as follows [2].

- Turn into transitions each control point (i.e., the start and the end of the boxes).

- Merge the transitions that represent control points that are executed at the same time.

- Add a sequence arc/place/arc between two transitions that have a temporal relation. Add the duration of the relation on the arc.
- The crossing condition of the transitions that represent control points with an interaction point is conditioned by receiving an external message. Moreover, the transition is fired automatically if the external event is not triggered between the interval of time specified by the composer.

Following the above steps, we translated the scenario shown in Figure 1 into its equivalent HTSPN structure (Figure 2). Here, the transitions with double stroke represent interaction points and the labels on the arcs define the duration of the relation. As mentioned above, the scenario is represented as a hierarchical box, then the relations $r 1$ and $r 2$ begin from the start of the scenario (i.e., transition $S(S)$ ). Additionally, the end of the scenario (i.e., transition $E(S)$ ) is defined by its duration (i.e., relation $r 9$ ), and the end of the boxes $C$ and $D$.

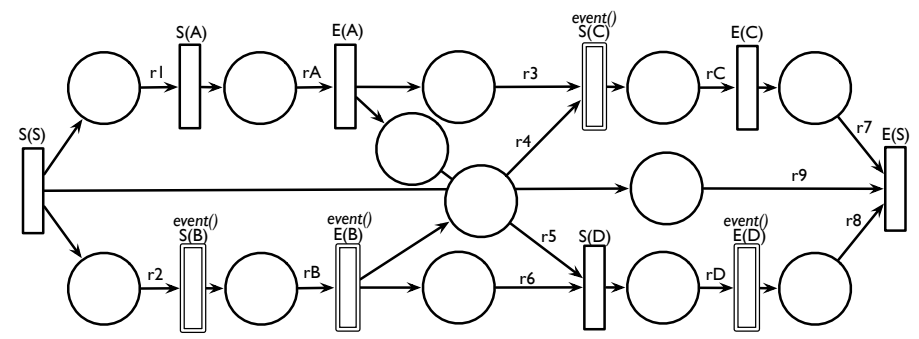

Fig. 2. HTSPN structure of the scenario shown in Figure 1.

\section{B. Coloured Petri Nets}

Coloured Petri Nets (CPNs) [4] is a graphical language for modelling and verifying concurrent and distributed systems. It combines the well-defined Petri Net model with the power of the high-level programming language, Standard ML.

CPN models can be simulated, then it allows to explore the possible behaviours of the system. In addition, CPN supports hierarchical composition which allows to structure a model into a set of modules. This feature is very important to model large systems. Another advantage of CPNs is the capability to express the notion of time. Next, we present the definition of Coloured Petri Net [4].

Definition 1: A timed non-hierarchical Coloured Petri Net is a nine-tuple $C P N_{T}=(P, T, A, \Sigma, V, C, G, E, I)$ where:

1) $P$ is a finite set of places.

2) $T$ is a finite set of transitions such that $P \cap T=\emptyset$.

3) $A \subseteq P \times T \cup T \times P$ is a set of directed arcs.

4) $\quad \Sigma$ is a finite set of non-empty colour sets. Each colour set is either untimed or timed.

5) $\quad V$ is a finite set of typed variables such that Type $[v] \in \Sigma$ for all variables $v \in V$.

6) $C: P \rightarrow \Sigma$ is a colour set function that assigns a colour set to each place. A place $p$ is timed if $C(p)$ is timed, otherwise $p$ is untimed.

7) $\quad G: T \rightarrow E X P R_{V}$ is a guard function that assigns a guard to each transition $t$ such that Type $[G(t)]=$ Bool.

8) $E: A \rightarrow E X P R_{V}$ is an arc expression function that assigns an arc expression to each arc $a$ such that

- $\quad$ Type $[E(a)]=C(p)_{M S}$ if $p$ is untimed; 
- $\quad$ Type $[E(a)]=C(p)_{T M S}$ if $p$ if timed. Here, $p$ is the place connected to the arc $a$.

9) $\quad I: P \rightarrow E X P R_{\emptyset}$ is an initialisation function that assigns an initialisation expression to each place $p$ such that

- $\quad$ Type $[I(p)]=C(p)_{M S}$ if $p$ is untimed;

- $\quad$ Type $[I(p)]=C(p)_{T M S}$ if $p$ is timed.

The verification of CPN models is automatic and supported by the state space method. Roughly, this method computes all reachable states and state changes of the CPN model and represents them as a directed graph. In this graph, nodes represent states and arcs represent occurring events. From the state space, it is possible to verify some properties of the system such as absence of deadlock.

CPN TooLs ${ }^{4}$ is a tool for editing, simulating and analysing CPNs models. This tool supports untimed and timed hierarchical CPN models. Therefore, we can build large and modular models. The user works directly with the graphical representation of the CPN model and the simulation can be carried out step by step or selecting the number of steps that will be executed. Also, we can define stop criteria and breakpoints. CPN TOOLS can generate full state space to verify models, and it uses advanced methods to mitigate the state explosion problem. This tool also supports model checking. An interesting feature is the generation of sophisticated reports from the states of the net during the simulation. Additionally, CPN ToOLs includes a collection of libraries for various purposes. One example is COMMS/CPN [6] that allows TCP/IP communication between CPN models and external applications.

\section{A MODEL FOR InTERACTIVE SCORES}

In this section we present a model for executing interactive scores [7] using Coloured Petri Nets (CPNs) [4]. This model allows to execute any scenario built in I-SCORE ${ }^{5}$.

As we explained before, in I-SCORE composers design a scenario defining a partial temporal organization of multimedia elements, represented as boxes, by using temporal relations. Additionally, composers can add interaction points to modify either the start date or the duration of the boxes during the execution. The temporal relations must maintain during the editing and execution time. Moreover, I-SCORE allows to group boxes in a hierarchical box. This is very important for designing large scenarios.

In our approach we develop a CPN model for each structure that defines an interactive score (i.e, temporal relations, boxes and interaction points). This allows to build a modular and parametrizable model which can be reusable and extensible. In the following we present the implemented CPNs modules.

\section{A. Intervals}

We model all elements of a score as intervals. These are classified into two classes: fixed intervals, and interactive intervals (Figure 3). Fixed intervals are temporal relations with a fixed duration, whereas interactive intervals have a range of values that define a minimum and a maximum duration.

\footnotetext{
${ }^{4}$ http://cpntools.org

${ }^{5}$ http://i-score.org/
}

Additionally, interactive intervals have attached an interaction point that stops the interval if it is triggered between the duration of the interval. We shall model boxes and interaction points from these two intervals.

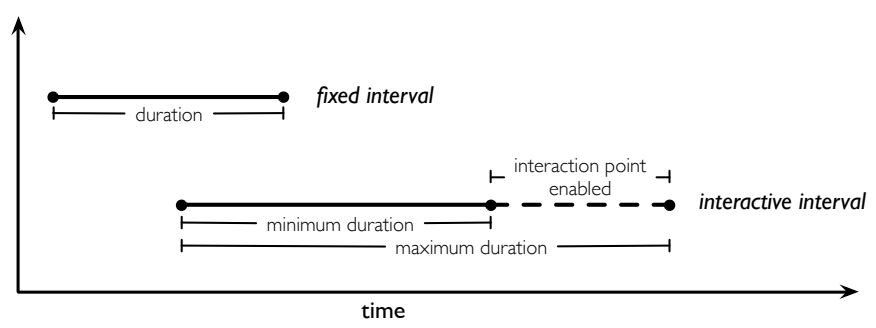

Fig. 3. Fixed and interactive intervals.

1) Fixed interval: A fixed interval consists in applying a delay between two points. This delay can be implemented in CPN using the delay expression $a+$ duration in the inscription of an output arc from a transition. As can be seen in Figure 4, if there is a token in the place start and a coloured token $d$ in the place duration, they will be consumed by the transition and a token will be produced in the place end after d time units.

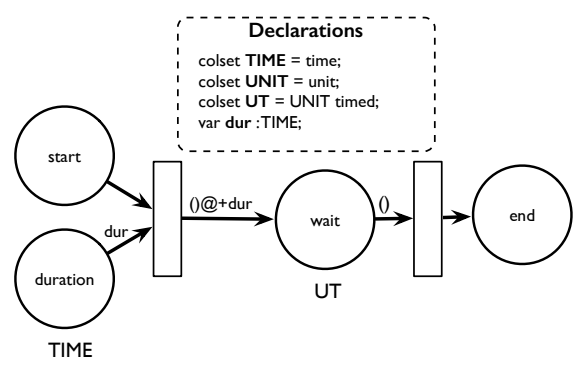

Fig. 4. CPN model of a fixed interval.

2) Interactive interval: An interactive interval applies a delay between two points whose duration is flexible (i.e., it has a minimum and a maximum duration). The interval finishes when either it reaches the maximum duration or the attached interaction point is triggered after the minimum duration. Therefore, we decompose an interactive interval into a module for modelling the flexible duration and other for handling the interaction point.

We use two fixed intervals for modelling flexible intervals. The first interval waits for the minimum duration of the flexible interval, and the second waits for the remaining time of the maximum duration (Figure 5). Then, once the minimum interval finishes, the second fixed interval starts and waits for the remaining time. We handle an interaction point using a net that accepts an event if it is both triggered after the start of the module and before that a stop signal, otherwise it is ignored (Figure 6). The module finishes if there is an accepted event or a stop signal. We use the guards of transitions in order to ignore the events (i.e., consume the tokens) that were not triggered in the current time. An inhibitor arc removes the conflict generated when there is a stop signal and an event at the same time. 


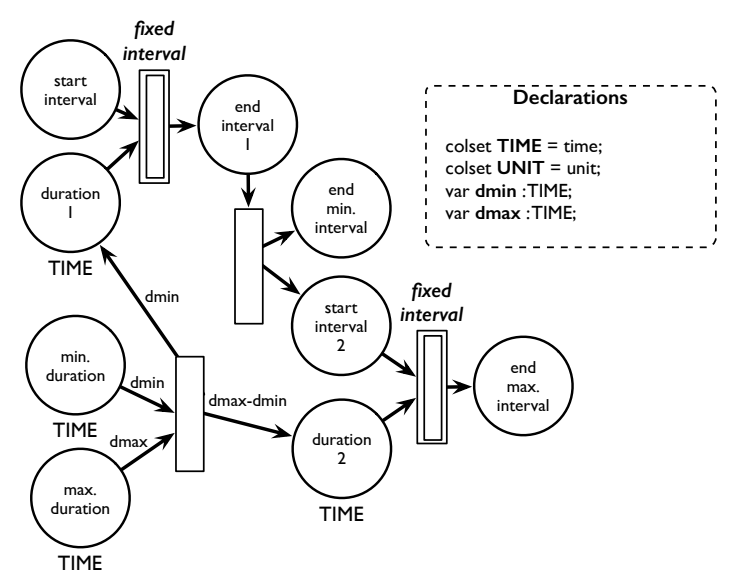

Fig. 5. CPN model of a flexible interval.

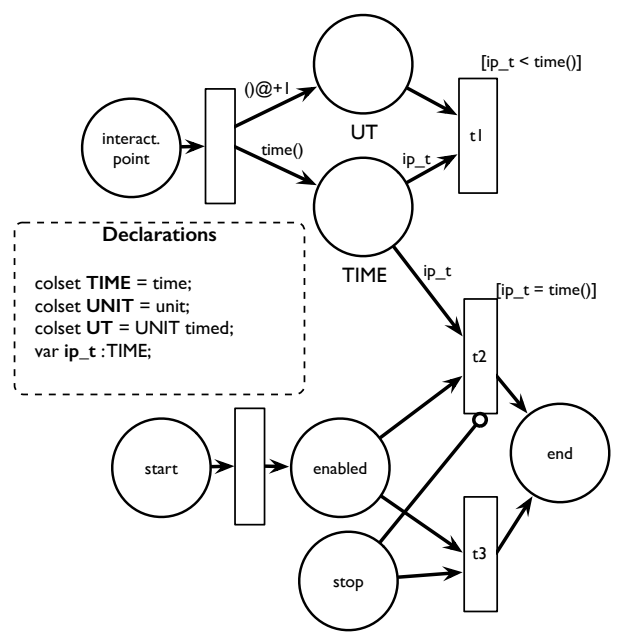

Fig. 6. CPN model for handling an interaction point.

Now, we use the above two modules to model interactive intervals (Figure 7). Therefore, we start the module for handling interaction points when the minimum duration is elapsed and we stop it when the flexible interval reaches the maximum duration. We limit the number of times that the module can be stopped (place "enable") if there are several intervals sharing the same interaction point. We shall discuss this in Subsection III-D.

\section{B. Boxes}

In I-SCORE, multimedia elements are temporal structures represented as boxes. A box has a duration and a start date which can be modified in the execution time by adding interaction points. The temporal properties of the scenario must be maintained during the edition and execution time. In our approach, we model boxes as fixed or interactive intervals with an attached process. Our models of the different cases of boxes are described below.

1) Fixed box: A fixed box is modelled as a fixed interval with a specific duration. In addition, the attached process starts

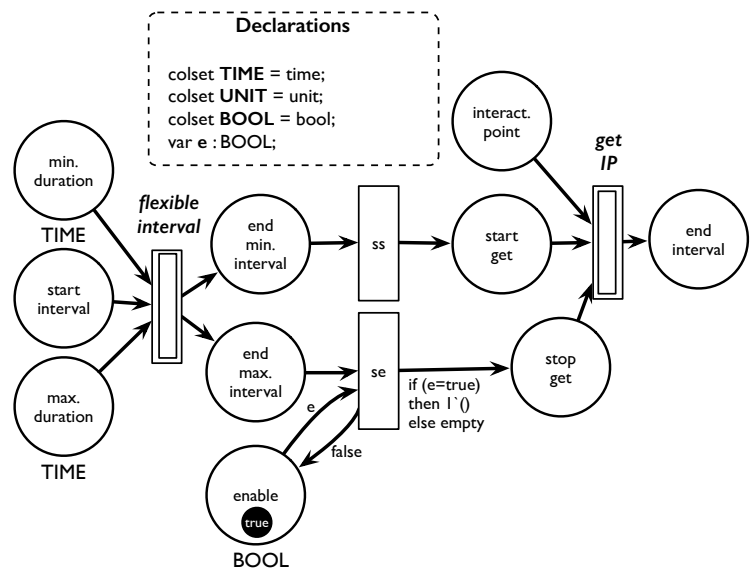

Fig. 7. CPN model of an interactive interval.

and finishes with the interval (Figure 8). We represent these events as transitions.

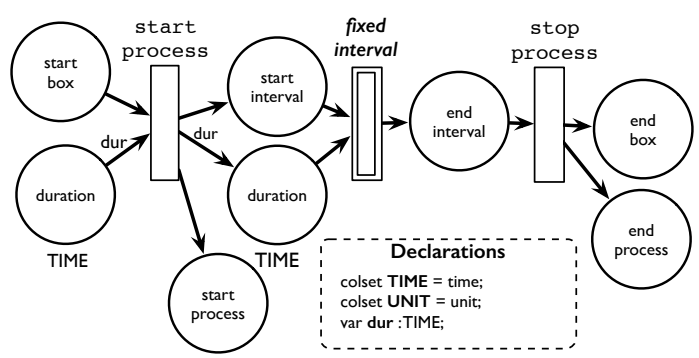

Fig. 8. CPN model of a fixed box.

2) Interaction point at the start of the box: An interaction point at the start of the box allows to anticipate or delay the start of the box during the execution. In I-SCORE, composers define an interval of time in which a specific event can be triggered. Then, the box will start if the event is triggered during the interval or the interval reaches its maximum duration. In our approach, we model this box adding an interactive interval at the start of a fixed box that controls the beginning of the box because it has attached an interaction point (Figure 9).

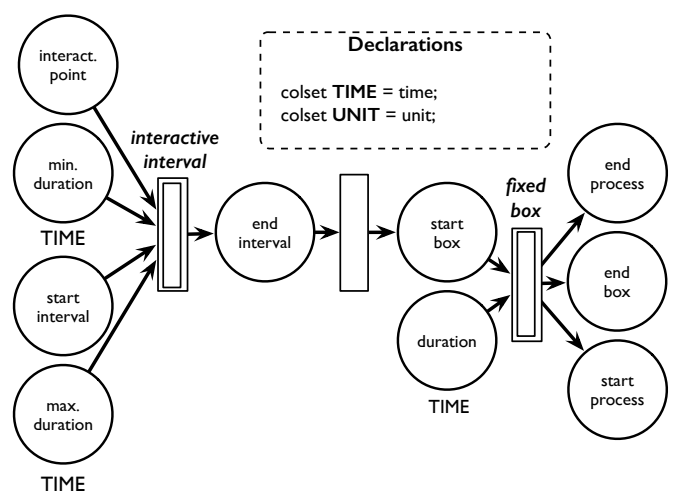

Fig. 9. CPN model of a box with an interaction point at the start. 
3) Interaction point at the end of the box: The duration of a box can be modified during execution if it has an interaction point at the end. Composers define an interval of time in which a particular event can be triggered. Then, the box will finish if the event is triggered during the interval or the box reaches its maximum duration. We model this box replacing the fixed interval of the fixed box (see Figure 8) with an interactive interval (Figure 10). The interactive interval handles the interaction point of the box and constraints the duration of the box.

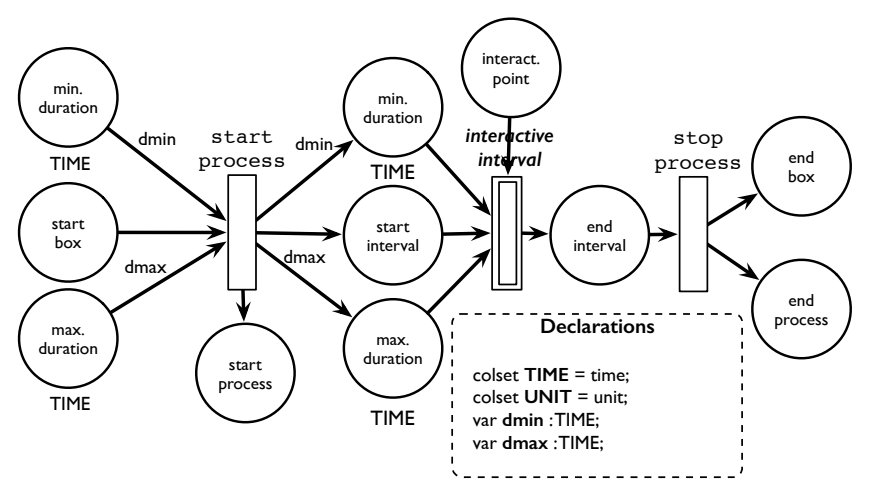

Fig. 10. CPN model of a box with an interaction point at the end.

4) Interaction point at the start and at the end of the box: In I-SCORE, composers can combine the two above behaviours. We take advantage of the modularity of our approach for modelling this box. Then, we only need to add an interactive interval before the start of a box with an interaction point at the end (Figure 11).

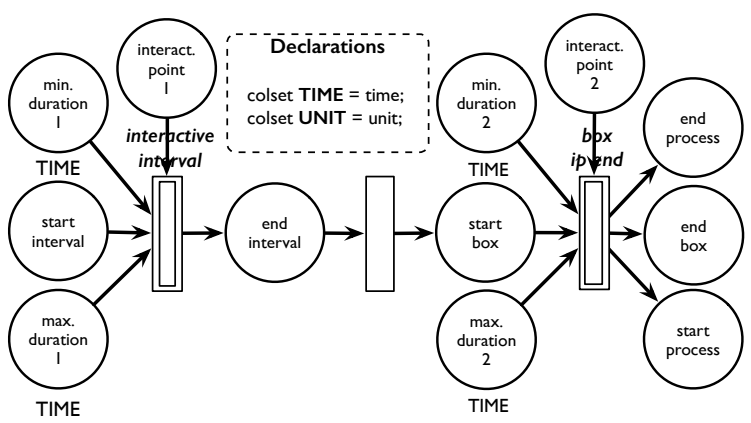

Fig. 11. CPN model of a box with an interaction point at the start and at the end.

\section{Hierarchy}

Hierarchy is very important for building complex scenarios. I-SCORE supports this feature allowing to group boxes in a hierarchical box. Indeed, the scenario is represented as a hierarchical box which contains all boxes, hierarchical boxes, temporal relations and interaction points. In our approach, a hierarchical box consists of two transitions for synchronizing the start and the end of its children. Furthermore, its duration depends on the temporal organization of the sub-scenario. We illustrate our idea in Figure 12.

Note that relations $A B$ and $A D$ start from the transition that represents the start of the hierarchical box. Then, these

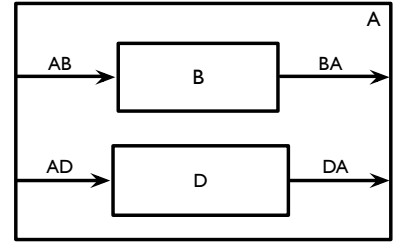

(a) Graphical representation

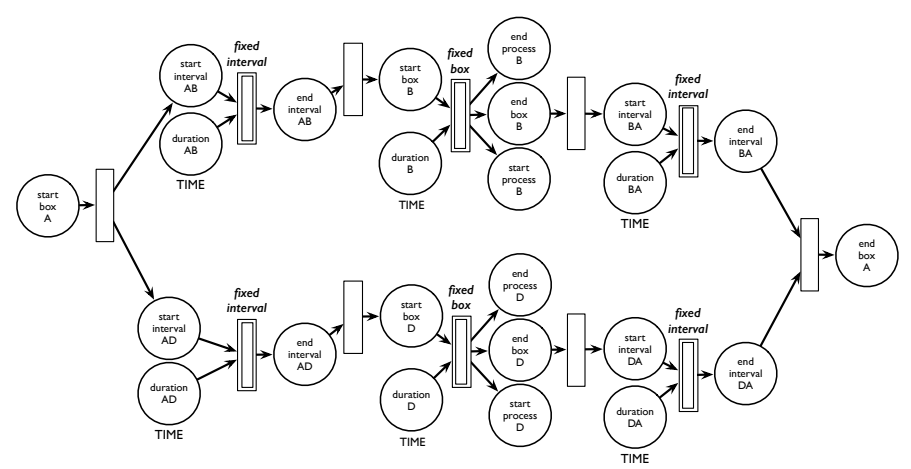

(b) CPN model

Fig. 12. Example of a hierarchical box with two children.

relations will start at the same time that box A. Moreover, observe that there is an arc between the end of the relations $B A$ and $D A$, and the transition that represents the end of the hierarchical box. Therefore, the hierarchical box $A$ will end when all the above relations finish.

In this paper, we only consider interaction points at the start of hierarchical boxes in order to simplify the presentation of the model. Interaction points at the end involve executing complex mechanisms to stop recursively the children of boxes.

\section{Synchronization}

Temporal relations define the beginning of boxes. Therefore, a box starts when all its preceding relations are satisfied. In I-SCORE, all relations preceding a box are flexible if the box has an interaction point at the start. Otherwise, they are fixed. In the following we introduce a merging operation to reduce several relations of the same type into an equivalent interval. The above allows to create a generic mechanism for maintaining the temporal relations in our model.

1) Merging fixed intervals: The fixed relations preceding a box must be fulfilled. Then, the box will start when all fixed intervals finish. We illustrate the above behaviour in Figure 13 that is the time-line of an execution of the scenario shown in Figure 1. In this scenario, the box $D$ has two preceding relations with fixed durations. Therefore, as can be seen in Figure 13, this box starts when the last interval finishes (i.e., interval $r 6$ at 16 seconds).

In our model, we merge several fixed intervals synchronizing their ends with the start of a box by using a transition (Figure 14). Then, the box will start when all its preceding relations have finished.

2) Merging interactive intervals: Temporal relations preceding a box with an interaction point are flexible (i.e., they have a minimum and a maximum duration). In that 


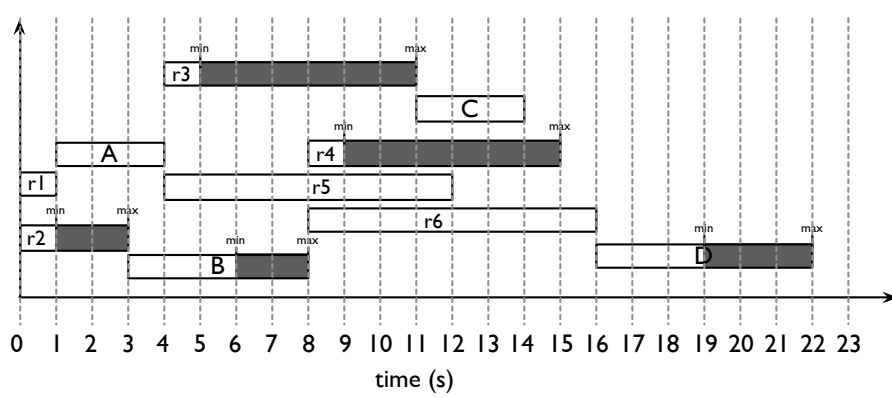

Fig. 13. Time-line of an execution of the scenario shown in Figure 1.

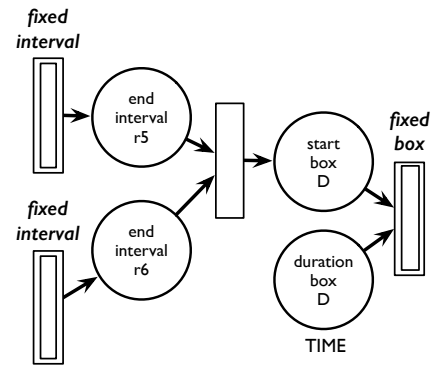

Fig. 14. CPN model for synchronizing fixed intervals.

case, the box will start when one of its preceding relations reaches its maximum duration or the event attached to the box is triggered after all relations have reached their minimum duration (Figure 15).

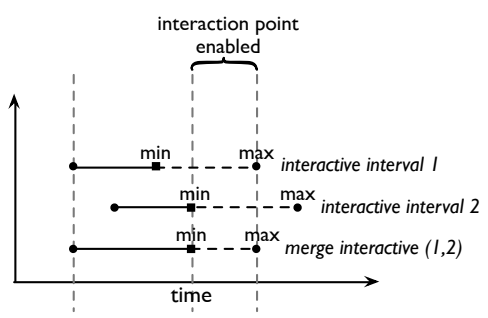

Fig. 15. Merging two interactive intervals.

We illustrate the above behaviour in Figure 16 that shows an execution of the scenario in Figure 1. In this scenario, box C has two preceding relations with flexible durations. Therefore, the box may start during the flexible interval generated by merging the relations $r 3$ and $r 4$. As can be seen in Figure 16, the box $\mathrm{C}$ started at 9 seconds because an event was triggered during the interval merge $(r 3, r 4)$. This interval represents the merging of the preceding intervals of the box, and it is defined by the minimum duration of the interval $r 4$ and the maximum duration of the interval $r 3$.

In our approach, we use interactive intervals to model temporal relations preceding a box with an interaction point. In order to merge several interactive intervals (Figure 17), we first split them into their two modules: flexible interval and interaction point (see Section III-A2). Then, we use a transition to synchronize the end of the minimum durations of all flexible intervals with the start of a module for handling the interaction point. Additionally, we connect the end of their maximum durations with the stop of the interaction point. As can be

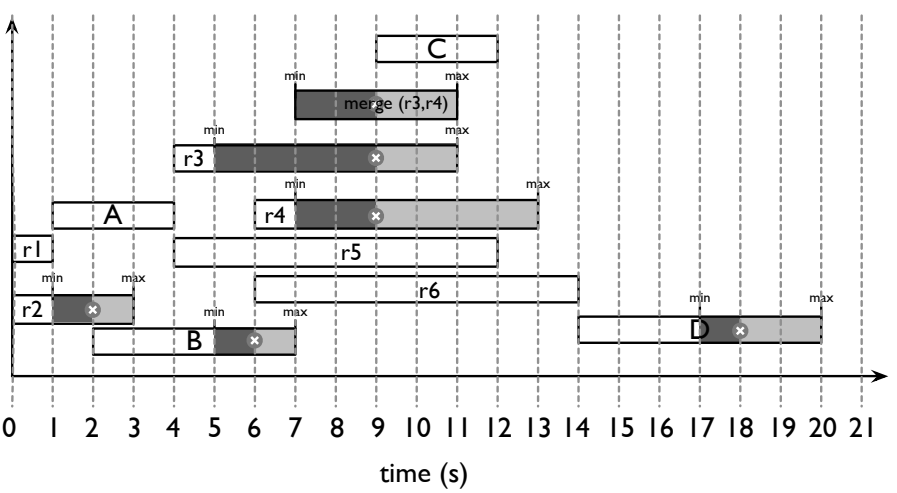

Fig. 16. Time-line of an execution of the scenario shown in Figure 2 with triggered interaction points. The symbol $\circledast$ represents a triggered event.

seen in Figure 17, we limit the number of times the interval can be stopped by using the place enable and the transition se.

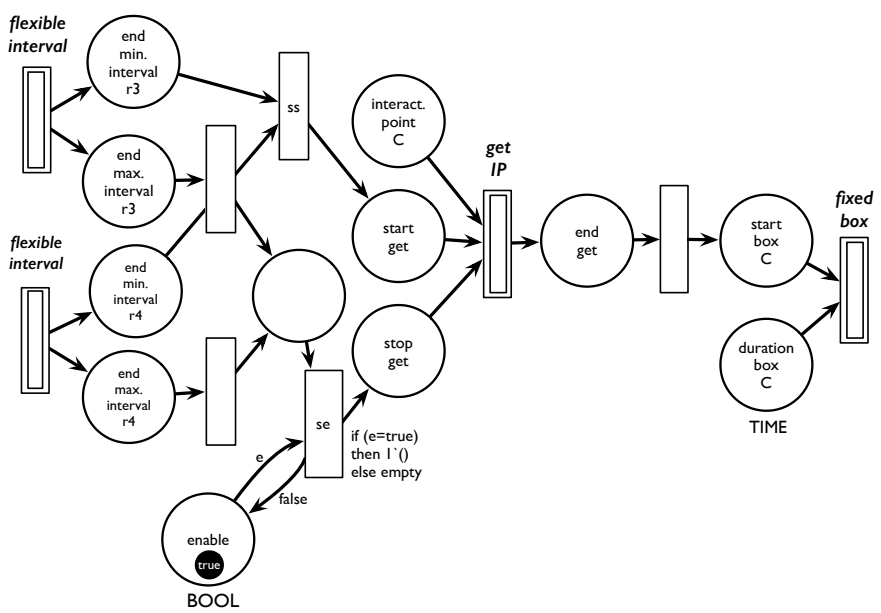

Fig. 17. CPN model for synchronizing interactive intervals.

Hence, the box will start when an interval reaches its maximum duration or the event attached to the box is triggered after all its preceding relations have reached their minimum durations.

\section{An Extension for Handling Complex Data}

In this section, we present an extension of the model described in Section III for handling complex data. We take advantage of the coloured tokens of CPNs to represent audio streams. In our approach, an audio stream consists of a set of tokens whose colour is a tuple $(i, d)$ where $i$ is the index of the audio frame and $d$ is its corresponding value. Additionally, the duration between each audio frame is preserved.

In the following we describe CPN modules for basic processing of audio files. First, we present a CPN model for reading audio files. Then, we show we can append and reverse files reusing the previous modules. Finally, we show the implementation and simulation of a scenario in CPN TOOLS. 


\section{A. Reading Audio Files}

In our approach, reading audio files consists in acquiring audio frames from a file with a determined frequency (i.e., duration between frames). We show the model of the above behaviour in Figure 18. Here, the transition READ gets an audio frame (i.e., a coloured token) from the file each time the interval of time finishes. The transition will continue to read the frames until it reaches the end of the file (i.e., the maximum number of frames in the file) or there is a stop signal.

Observe that the inhibitor arc allows to solve the conflict generated when a stop signal is present at the same instant that a frame can be read. In addition, the place limit restricts the module to read a new frame only if the previous one was completely processed (i.e., the token is in the place output), and the place stop_enabled disables the stop transition when the module has finished.

The module has as output the read frames (place output) and the number of read frames (place frames_read). Moreover, it also indicates if the module reached the end of the file (place $\mathrm{EOF}$ ), and the instant when the interval of time between frames is elapsed (place next_frame). The last feature is useful if we want to synchronize the output of two files.

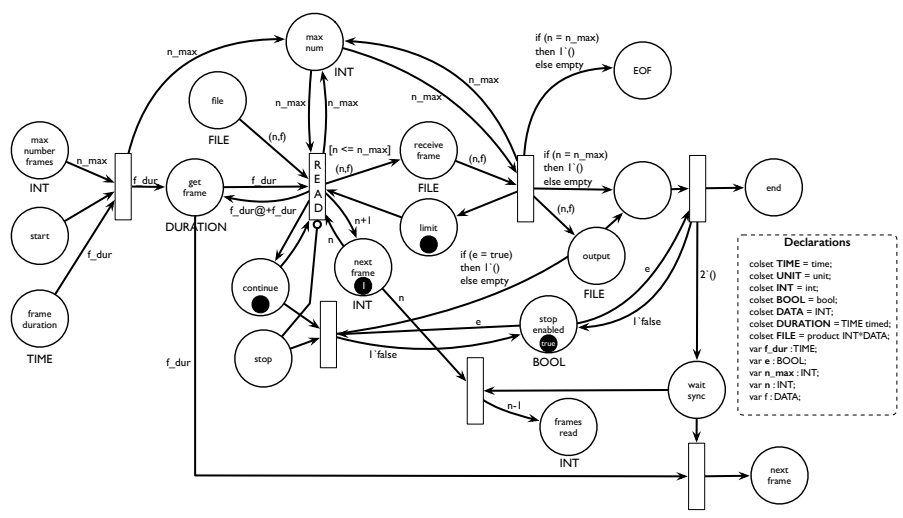

Fig. 18. CPN model for reading a file.

\section{B. Appending Files}

We append two files concatenating their outputs. We took advantage of the modularity of our model to build this module using the above. As can be seen in Figure 19, the module reads the first file, and once it finishes, it synchronizes the start of the reading of the second file. That means that the first frame of the second file will be read when the duration between the frames of the first file is elapsed (i.e., there is a token in the place next_frame).

Observe that the concatenated file is stored in the place output. We add an offset to the indices of the second file to correctly build the concatenated file (place index). Therefore, the outputs of the module are the appended file (place output) and the number of concatenated frames (place total_frames_read). The module will finish if both files are successfully read or there is a stop signal.

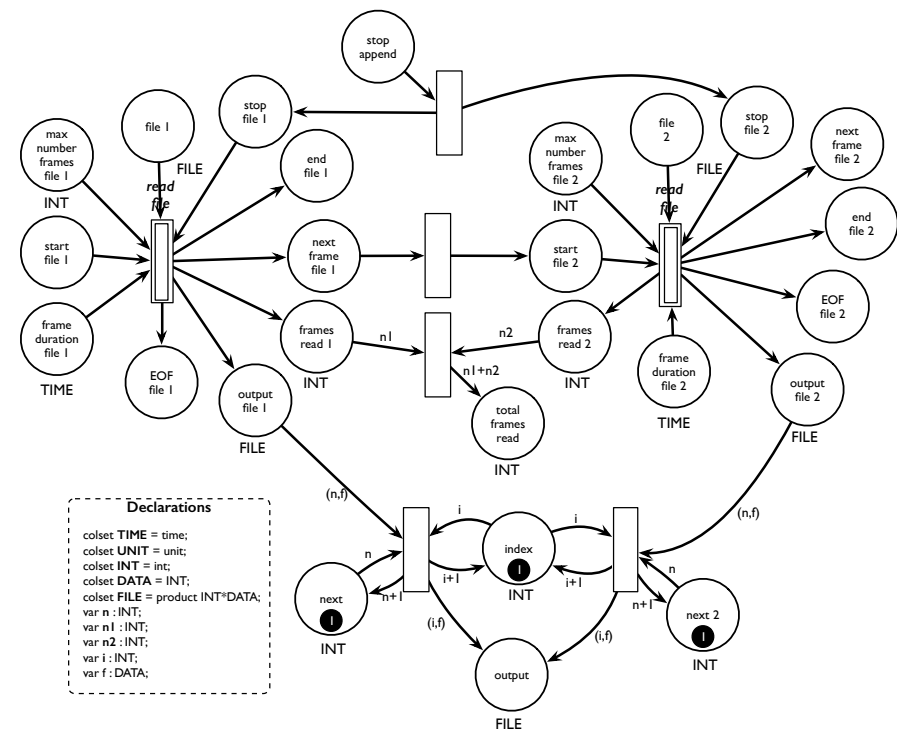

Fig. 19. CPN model for appending files

\section{Reversing Files}

In our approach, the module reverses the order of a file in one instant of time, and then reads the output. As can be seen in Figure 20, we make use of the module for reading files to read the file in one time (i.e., the duration between frames is zero) while the transition REV reverses the order of its indices. Once the order of the whole file is inverse (i.e., the index of the reversed file is zero), another module starts to read the inverse file with a specific frequency. Observe that this module has the same outputs than the module for reading files.

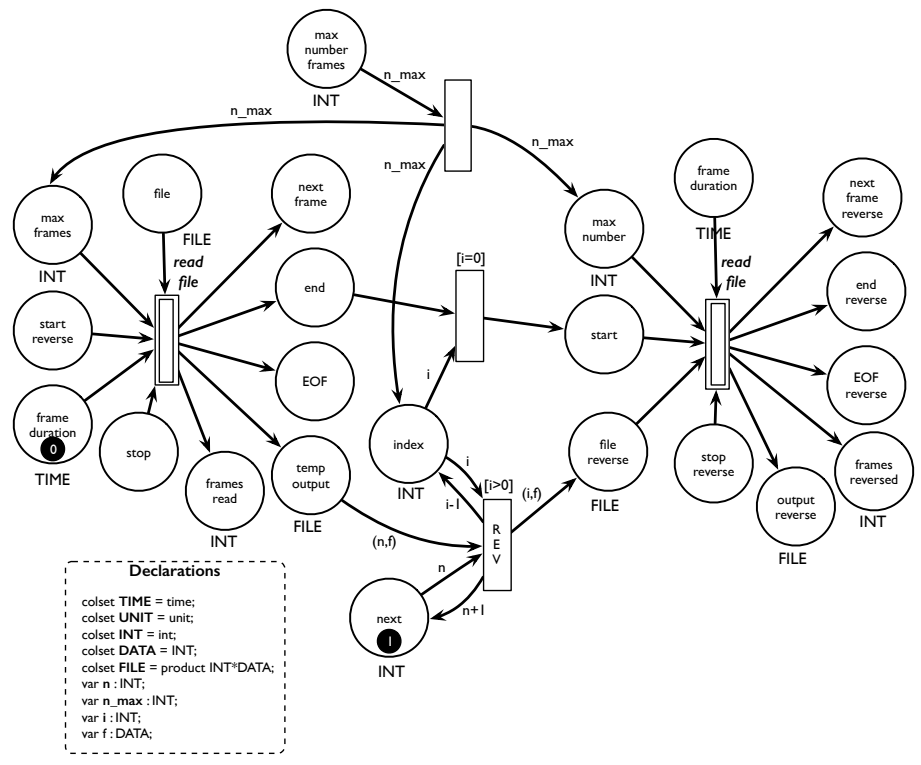

Fig. 20. CPN model for reversing a file. 


\section{Simulation}

In the following we illustrate the use of the modules presented in this section by means of the scenario shown in Figure 21. Roughly speaking, the scenario first reads two files. Then, a box for reversing files receives, as input, the read frames of the second file to reverse them. Finally, a box for appending files receives, as input, the reversed file and the first file to concatenate them.

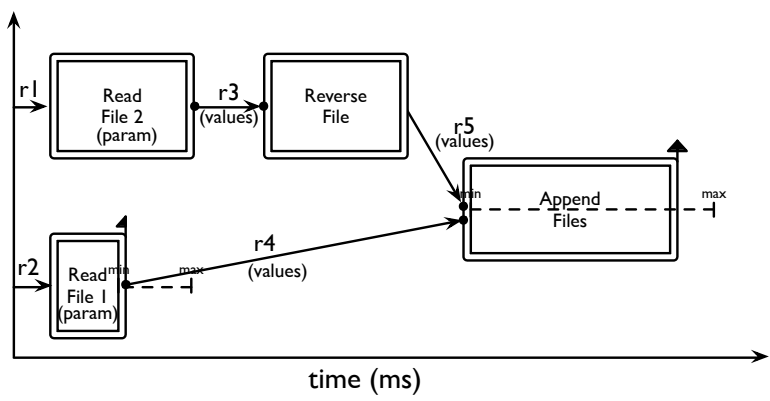

Fig. 21. Scenario with data processing.

As can be seen, the data processing modules are organized by using the elements described in Section III. For the sake of simplicity, we mixed boxes with processing modules in a single box. Additionally, we added to boxes inputs and outputs which are represented as points on each edge of the box. Intervals allow to communicate values between boxes and they are used as parameters of processes (e.g., number of frames, duration between frames). For example, the box for reversing files receives, as input, the output of the box for reading files through the interval $r 3$.

Moreover, the duration of boxes can be constrained with the duration of their processes by adding an interaction point at the end of the box whose associated event is triggered when the process finishes. Observe that we use a different symbol in the box for appending files to represent the new interaction point.

Let us illustrate in Figure 22 a possible execution of the above scenario assuming the following configuration.

- $\quad$ The first file is composed of 4 frames, and each frame must be played every 3 milliseconds.

- The second file is composed of 5 frames, and each frame must be played every 3 milliseconds.

- $\quad$ Both files start to be read once the scenario begins.
- The reading of the first file must stop in 9 milliseconds. However, it can be stopped by the performer after 6 milliseconds.

- The reading of the second file must stop in 13 milliseconds.

- Once the reading of the second file finishes, the scenario must wait a millisecond, and then the read file is reversed. This box must stop in 13 milliseconds.

- $\quad 3$ milliseconds after of having read the first file and 5 milliseconds after of having reversed the second file, both files are concatenated. This box must finish in 25 milliseconds or when its attached process finishes.

Note that, if the box for reading the first file is stopped at 7 milliseconds by triggering its interaction point, only 3 frames of the file are read. On the other hand, the second file is completely read and reversed. Additionally, when the process for appending files finished (at 53 milliseconds), it triggered the event for stopping the corresponding box. Therefore, at the end of execution, the concatenated file is composed of 8 frames; 3 frames from the first file and 5 frames from the second file.

We conclude this section with a report showing the simulation in CPN TOOLS of the above scenario. Here, we assume that each time unit of the simulator tool represents a millisecond. As can be seen in Figure 22, we use boxes and intervals to define the temporal organization of the scenario. Furthermore, each process is initialized with its inputs (e.g., the number of frames, the duration between frames), but during execution some parameters will be passed by other boxes through intervals (e.g., the file that will be reversed).

Now, we emphasize the main events generated in the report. At 7 milliseconds the box for reading the first file was stopped by the performer, then only 3 frames of the file were read. Observe that each frame was processed preserving the corresponding delay (i.e., 3 milliseconds). At 12 milliseconds the second files was completely read, but the box finished at 13 milliseconds. Note that the output of a process is buffered in order to be read later by another process. At 14 milliseconds, the second file started to reverse and finished at 26 milliseconds. At 32 milliseconds all preceding intervals of the box for appending files were satisfied and the box started to concatenated the first file and the reversed second file. Finally, the process finished at 53 milliseconds, then the box also was stopped at the same time. Notice that the output of the above process corresponds correctly to the transformations applied to files read at the beginning of the scenario.

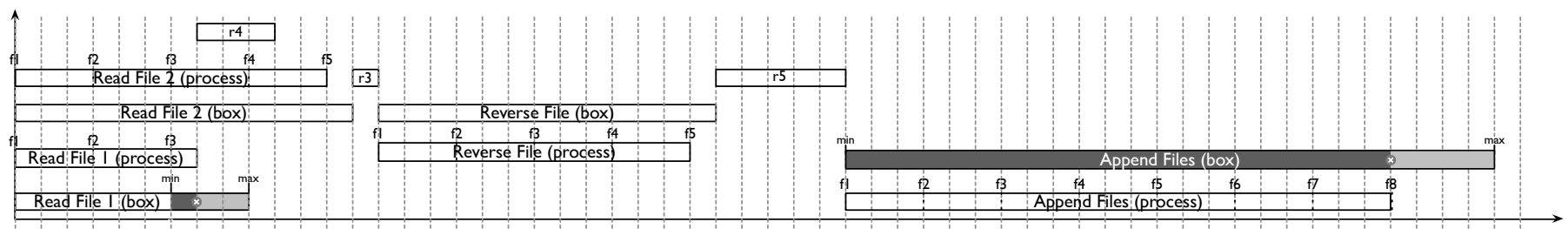

$0 \quad 1 \quad 2 \quad 3 \quad 4 \quad 5 \quad 6 \quad 7 \quad 8 \quad 910111213141516171819202122232425262728293031323334353637383940414243444546474849505152535455565758$ time (ms)

Fig. 22. Time-line of an execution of the scenario shown in Figure 21. The symbol $\circledast$ represents a triggered event. 
Hence, comparing the simulation report in Figure 22 with the execution time-line in Figure 22, we observe that our model executed correctly the scenario and preserved the temporal organization of the score during execution. Moreover, it follows the execution model of I-SCORE and also allows to handle complex data such as audio streams.

\section{CONCLUding REMARKS}

In this paper we presented a model to execute interactive scores [1] with the ability to handle complex data, in particular, data audio streams. We used Coloured Petri Nets [4] to model audio frames as coloured tokens that are handled by processes. These processes have inputs and outputs, and they can pass values between them through intervals. The above allows the functional composition of processes. We took advantage of this functional composition and the hierarchy support of CPNs to build a modular model. Thus, we extended the model of the ISCORE software with modules for processing audio files such as reading, appending and reversing files.

We illustrated the notion of asynchronous functional composition of our model by simulating the scenario shown in Figure 21. Here, the outputs of the modules for reversing and appending files are buffered before the module for appending files starts. Also, we implemented a method for merging intervals which allows to translate any temporal property defined by the user into our model. We implemented our model in CPN TOOLS, and then we simulated a scenario that allows to observe that our approach models interactive scores and audio streams correctly.

Future Work. Interactive scores have a wide range of applications in all types of industries, for example, video games, live performance installations, and virtual museum visits [8]. The use of loops and conditionals are necessary for the proper design of scenarios for these kind of applications. However, the model of I-SCORE does not support them. We plan to take advantage of the capability of our model for handling complex data and the power of the CPN formalism to model these features. In a first approach, we could represent conditionals like the switch statement of the $\mathrm{C}$ programming language where: each case could be the guard of a set of transitions that have associated a sub-scenario; and a coloured token that carries the value that will be compared. Therefore, the transition whose guard is fulfilled with the value of the token will execute the corresponding sub-scenario. We could represent loops like a hierarchical box that executes its children until a specific condition is false. Note that, the implementation of loops requires conditionals.

Additionally, audio processing is very important in the applications listed above. Then, we plan to extend our model with new audio processing processes. For example, we could change the play speed of an audio by modifying the interval of time in which the module for reading files reads each frame. Also, we could amplify the audio volume by scaling the values of each read frame.

Finally, we plan to verify properties about scenarios like the maximum number of processes that can be executed at the same time in a specific machine, the maximum duration of the buffers, the maximum duration of the scenario, among others.

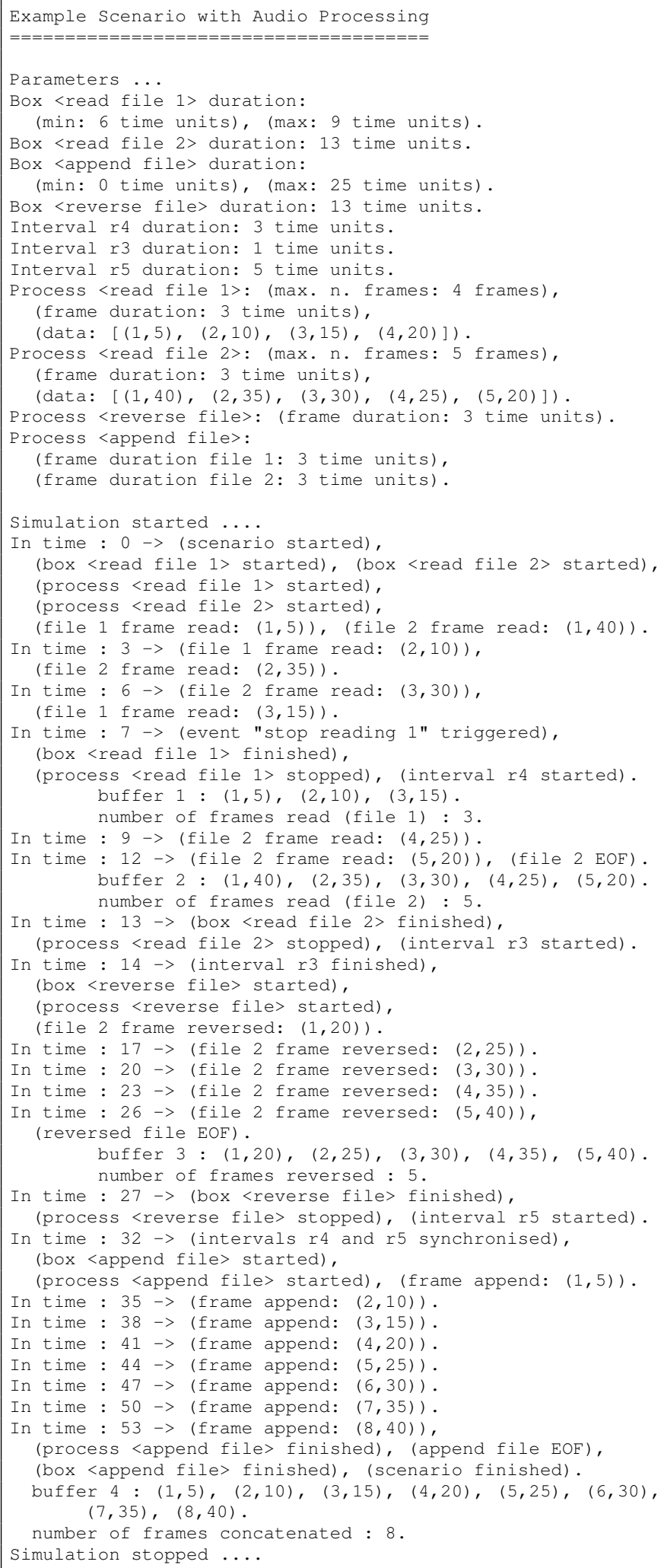

Fig. 22. Simulation report of an execution of the score shown in Figure 21.

\section{ACKNOWLEDGMENTS}

We thank the anonymous reviewers for their detailed comments that helped us to improve this paper. This work has been 
supported by the OSSIA (ANR-12-CORD-0024) project and SCRIME ${ }^{6}$.

\section{REFERENCES}

[1] A. Allombert, "Aspects temporels d'un système de partitions musicales interactives pour la composition et l'exécution."

[2] R. Marczak, M. Desainte-Catherine, and A. Allombert, "Real-time temporal control of musical processes," in The Third International Conferences on Advances in Multimedia, ser. MMEDIA 2011, pp. 12-17.

[3] P. Sénac, P. de Saqui-Sannes, and R. Willrich, Hierarchical Time Stream Petri Net: A Model for Hypermedia Systems, ser. Lecture Notes in Computer Science. Springer Berlin Heidelberg, vol. 935, pp. 451-470.

[4] K. Jensen and L. M. Kristensen, Coloured Petri Nets. Modelling and Validation of Concurrent Systems. Dordrecht; New York: Springer, 2009.

[5] J. F. Allen, "Maintaining knowledge about temporal intervals," vol. 26, p. $832-843$.

[6] G. Gallasch and L. M. Kristensen, "Comms/CPN: a communication infrastructure for external communication with Design/CPN," in Third Workshop and Tutorial on Practical Use of Coloured Petri Nets and the CPN Tools. DAIMI PB-554, pp. 75-91.

[7] M. Desainte-Catherine, A. Allombert, and G. Assayag, "Towards a hybrid temporal paradigm for musical composition and performance: The case of musical interpretation," Computer Music Journal, vol. 37, no. 2, pp. 61-72, 2013.

[8] A. Allombert, M. Desainte-Catherine, and G. Assayag, "Iscore: a system for writing interaction," ser. DIMEA '08. Athens, Greece: ACM, Sep. 2008 , p. $360-367$

${ }^{6} \mathrm{http} / / / \mathrm{scrime}$. labri.fr 